\title{
Podiatric habits in people with nail disorders
}

\author{
Abián Mosquera-Fernández1, María Díaz-Rodríguez², Cristina González-Martín, Marta Platero-Arboiro², \\ Victoriano Souto-Rey ${ }^{4}$ and Vanesa Balboa-Barreiro ${ }^{3}$ \\ ${ }^{1}$ Department of Health Sciences, Faculty of Infirmary and Podiatry, Universidade da Coruña (UDC); ${ }^{2}$ Podiatry Degree, UDC; ${ }^{3}$ Clinical Epidemiology \\ Research Group, Department of Health Sciences, Faculty of Infirmary and Podiatry, UDC; ${ }^{4}$ Department of Orthopedic Surgery and Traumatology, \\ Complejo Hospitalario Universitario de Ferrol (Área Sanitaria de Ferrol, España).
}

\begin{abstract}
Objective: To determine the prevalence of onychocryptosis and onychomycosis confirmed by culture in subjects attending a Podiatric University Hospital and to describe their podiatric habits. Method: Cross-sectional study of prevalence. The study was carried out at the Clinic University of Podology of A Coruna University (Ferrol, Spain). Review of 1082 clinical histories, of which 170 were selected, persons who had onychocryptosis diagnosed clinically. The presence of onychomycosis confirmed by cultivation, presence of onychocryptosis through clinical diagnosis and a questionnaire was handed out of podologic habits. Results: Mean age of the total sample $(n=1082)$ was $47.9 \pm 22.1$ years. Prevalence of onychocryptosis was $15.7 \%(n=170)$ with a mean age of $54.1 \pm 20.1$ years and with a significantly higher affectation in women and people under 65 . Prevalence of onychomycosis was $17.6 \%(n=30)$, mean age $59.5 \pm 20.5$ years with greater involvement in women and people over 65 years. With regard to the questionnaire of habits, the footwear more employee was the closed, cordoned off and heel flat. The majority of the subject came to a podiatrist for the first time; walked approximately 1 hour and their daily activity made of foot with short displacements. Conclusions: A high prevalence of onychocryptosis and onychomycosis in people attending Podiatric services has been found. For its part using a type of flat or low heel and who does the care of toenails are shown as predictive variables of onychomycosis.
\end{abstract}

KEY WORDS: Foot care. Foot disease. Podiatric habits. Onychocryptosis. Onychomycosis. Nail pathology.

\section{Introduction}

Podiatric disorders constitute a highly prevalent problem in the population, especially in people older than 65 years ${ }^{1}$. In this sense, Munro and Steele ${ }^{2}$ refer that $70 \%$ of people older than 65 years suffer from some type of foot problem. Recently, claw toes $(69.7 \%)$ and hallux valgus (38\%) have been observed to be most common podiatric alterations in people older than 40 years in Spain ${ }^{3}$. Secondarily, both conditions can act as etiologic factors for onychocryptosis ONC), a pathology that often is accompanied by pain, inflammation and infection that, in case of becoming chronic, elicits the appearance of granulation tissue that covers the nail groove and major complications such as osteomyelitis ${ }^{4-6}$. ONC is variable, and although there is no unanimity with regard to which gender is more affected, most authors agree that it occurs more commonly in adolescents and young adults on the second and third decades of life $e^{9,10}$. At the same time, it is considered to be more prevalent in the first toe, with the peroneal canal being more affected, at a 2:1 ratio with regard to the tibial canal. In turn, the incidence of onychomycosis (ONM) has experienced an increase in the past few years owing mainly to population aging. It accounts for between 18 and $40 \%$ of nail disorders, and its prevalence ranges from 2 to $18 \%$ among the world population $n^{7,11-13}$. Both pathologies (ONC and ONM) are highly common nail

\section{Correspondence:}

Gac Med Mex. 2017;153:733-740

Contents available at PubMed www.gacetamedicademexico.com 
disorders seen in the daily podiatric consultation, with ONC being observed to be associated with onychomycotic infections to a greater extent from the third decade of life on, although it can also occur as a complication of oral antifungal therapy $y^{4,7,14-16}$.

\section{Method}

A cross-sectional study was carried out at the Podiatry University Clinic of the Universidade da Coruña (Ferrol, Spain) by means of the review of the medical histories of people attending an appointment for the first time and for any reason between September 2012 and September 2014. Around 1000 people were estimated would be attending an appointment during that period, which is a sample size that allows knowing the characteristics presented by these people with regard to the pathologies to be studied (ONC and ONM) with a $95 \%$ confidence interval and an accuracy of $\pm 3 \%$. Data were registered by a single clinician, with all subjects that granted freely and voluntarily their written consent being included. People with amputation of any of their lower extremities, people with suspected ONM but with negative cultures and all those not granting written informed consent were excluded, with final sample comprising 1082 reviewed medical histories. Sociodemographic (age and gender) and clinical (presence of ONC) variables were collected from each medical history. In addition, from the 1082 reviewed histories, those people with clinically diagnosed ONC were selected in order to collect information regarding the podiatric habits questionnaire that as per protocol is completed during the first visit (Fig. 1), as well as to determine the culture-confirmed presence of ONM.

The study was approved by the Research Ethics Committee of the Galician Heath Service (Xunta de Galicia, Spain), with registration code 2015/113. At all times, the ethical standards picked up by international regulations for epidemiological studies and biomedical research in human subjects ethical evaluation were followed, and patient anonymity was ensured by obeying the Law for the Protection of Personal Data (Organic Law 15/1999 of December 13), the Law $41 / 2002$ (basic regulatory of patient autonomy and of rights and obligations in matters of clinical information and documentation), the Law 3/2001 (regulatory of patient informed consent and medical history) and the Law 3/2005, which is a revision of Law 3/2001.

The information was analyzed with the IBM SPSS ${ }^{\circledR}$ clinical package (version 20.0) for Windows ${ }^{\circledR}$, and a descriptive analysis of the variables included in the

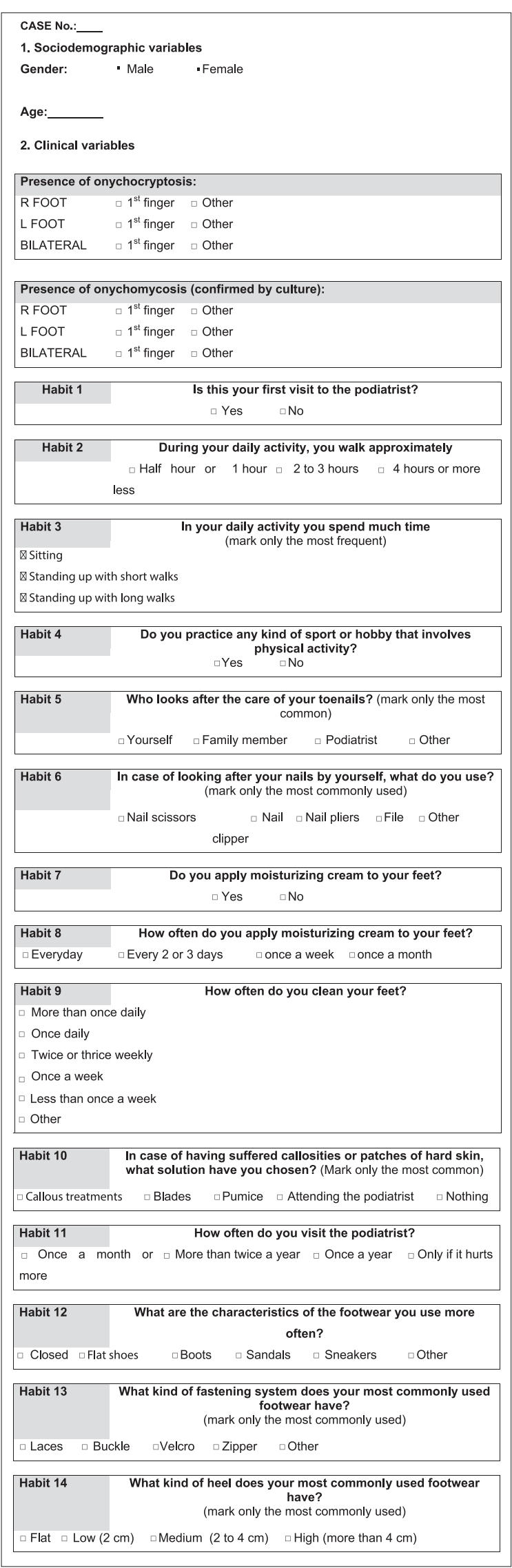

Figure 1. Podiatric habits questionnaire. 
Table 1. Patient description according to age, gender and number of onychocryptosis and onychomycosis localizations

\begin{tabular}{|c|c|c|}
\hline & $\begin{array}{l}\text { Onychocryptosis } \\
\qquad(n=170)\end{array}$ & $\begin{array}{l}\text { Onychomycosis } \\
(n=30)\end{array}$ \\
\hline & Mean \pm SD & Mean \pm SD \\
\hline Age & $54,09 \pm 20,09$ & $58,57 \pm 19,86$ \\
\hline \multirow[t]{2}{*}{ Number of localizations } & $1.56 \pm 0.62$ & $1.2 \pm 0.41$ \\
\hline & n (\%) & n (\%) \\
\hline \multicolumn{3}{|l|}{ Gender } \\
\hline Male & $49(28.8)$ & $11(36.7)$ \\
\hline Female & $121(71.2)$ & $19(63.3)$ \\
\hline \multicolumn{3}{|l|}{ Localizations } \\
\hline \multicolumn{3}{|l|}{ Right foot } \\
\hline First toe & $127(74.7)$ & $16(9.4)$ \\
\hline Other toes & $8(4.7)$ & $2(6.7)$ \\
\hline \multicolumn{3}{|l|}{ Left foot } \\
\hline First toe & $122(71.8)$ & $17(10)$ \\
\hline Other toes & $9(5.3)$ & $1(3.3)$ \\
\hline Both feet first toe & $83(48.8)$ & $5(16.7)$ \\
\hline
\end{tabular}

study was carried out. Quantitative variables were expressed as the mean, and qualitative variables, as absolute values (n) and percentages. To compare the quantitative variables' means with regard to dichotomous qualitative variables, Student's t-test or Mann-Whitney test were used as appropriate after quantitative variables normality was verified with the Kolmogorov-Smirnov test. Qualitative variables association was estimated by means of the chi-square test and, in turn, the risk for the event to occur was estimated by calculating the odds ratio and its $95 \%$ confidence interval.

\section{Results}

General characteristics of the studied sample are presented in table 1, where it can be appreciated that mean age of the 170 participants with ONC is 54.09 years $( \pm 20.09)$, and that the female gender is predominant $(71.2 \%$ vs. $28.8 \%)$. Mean number of sites in these patients is $1.56( \pm 0.62)$, with both left and right foot first toe localizations being predominant (71.8\% vs $74.7 \%)$. Sites with this pathology in both feet are presented by $48.8 \%$ of people. Of the 170 subjects with ONC, 30 have ONM (17.6\%), with a mean age of 58.57 years $( \pm 19.86)$ and female gender predominance as well $(63.3 \%$ vs. $36.7 \%)$.

Mean number of sites with ONM in these patients is $1.2( \pm 0.41)$, with both left and right foot localizations similarly predominating $(9.4 \%$ vs. $10 \%)$.
Table 2. Patient description according to their habits by type of pathology

\begin{tabular}{|c|c|c|}
\hline \multirow[t]{2}{*}{ Habits } & $\begin{array}{l}\text { Onychocryptosis } \\
(n=170)\end{array}$ & $\begin{array}{l}\text { Onychomycosis } \\
\qquad(n=30)\end{array}$ \\
\hline & $\mathrm{n}(\%)$ & $\mathrm{n}(\%)$ \\
\hline \multicolumn{3}{|l|}{1} \\
\hline No & $68(47.2)$ & $12(50.0)$ \\
\hline Yes & $76(52.8)$ & $12(50.0)$ \\
\hline \multicolumn{3}{|l|}{2} \\
\hline Less than half an hour & $51(30.0)$ & $11(44.0)$ \\
\hline 1 hour & $58(34.1)$ & $8(32.0)$ \\
\hline Between 2 and 3 hours & $20(11.8)$ & $3(12.0)$ \\
\hline 4 or more hours & $12(7.1)$ & $3(12.0)$ \\
\hline \multicolumn{3}{|l|}{3} \\
\hline Sitting & $43(25.3)$ & $8(32.0)$ \\
\hline Standing up/short walks & $76(44.7)$ & $15(60.0)$ \\
\hline Standing up/long walks & $27(15.9)$ & $2(8.0)$ \\
\hline \multicolumn{3}{|l|}{4} \\
\hline No & $34(36.9)$ & $5(35.7)$ \\
\hline Yes & $58(63.1)$ & $9(64.3)$ \\
\hline \multicolumn{3}{|l|}{5} \\
\hline Yourself & $107(71.3)$ & $21(80.8)$ \\
\hline Family member & $20(13.3)$ & 0 \\
\hline Podiatrist & $15(10.0)$ & $5(19.2)$ \\
\hline Other & $11(7.3)$ & $2(7.7)$ \\
\hline \multicolumn{3}{|l|}{ 5-rec } \\
\hline Yourself or a professional & $121(80.7)$ & $25(96.2)$ \\
\hline Non-professional & $29(19.3)$ & $1(3.8$ \\
\hline \multicolumn{3}{|l|}{6} \\
\hline Nail scissors & $43(28.5)$ & $6(23.1)$ \\
\hline Nail clipper & $57(37.7)$ & $9(34.6)$ \\
\hline Nail pliers & $38(25.2)$ & $8(30.8)$ \\
\hline File & $21(13.9)$ & $2(7.7)$ \\
\hline \multicolumn{3}{|l|}{7} \\
\hline No & $82(55.8)$ & $17(70.8)$ \\
\hline Yes & $65(44.2)$ & $7(29.2)$ \\
\hline \multicolumn{3}{|l|}{8} \\
\hline Everyday & $22(31.4)$ & $3(42.9)$ \\
\hline Every 2-3 days & $25(35.7)$ & $1(14.3)$ \\
\hline Once a week & $17(24.3)$ & $1(14.3)$ \\
\hline Once a month & $6(8.6)$ & $2(28.6)$ \\
\hline \multicolumn{3}{|l|}{9} \\
\hline More than once daily & $29(20.3)$ & $2(8.7)$ \\
\hline Once daily & $78(54.5)$ & $15(65.2)$ \\
\hline 2-3 times per week & $31(21.7)$ & $4(17.4)$ \\
\hline Once weekly & $4(2.8)$ & $2(8.7)$ \\
\hline Other & $1(0.7)$ & 0 \\
\hline \multicolumn{3}{|l|}{10} \\
\hline Callous treatments & $11(9.0)$ & 0 \\
\hline Blades & $18(14.8)$ & $1(5.0)$ \\
\hline Pumice stone & $36(29.5)$ & $5(25.0)$ \\
\hline Visit the podiatrist & $31(25.4)$ & $5(25.0)$ \\
\hline Nothing & $30(24.6)$ & $7(35.0)$ \\
\hline \multicolumn{3}{|l|}{11} \\
\hline Once monthly & $11(19.3)$ & $1(3.3)$ \\
\hline
\end{tabular}


Table 2. Patient description according to their habits by type of pathology (Continued)

\begin{tabular}{|c|c|c|}
\hline \multirow[t]{2}{*}{ Habits } & $\begin{array}{l}\text { Onychocryptosis } \\
(n=170)\end{array}$ & $\begin{array}{l}\text { Onychomycosis } \\
(n=30)\end{array}$ \\
\hline & n (\%) & n (\%) \\
\hline More than twice yearly & $9(15.8)$ & $4(40.0)$ \\
\hline Once yearly & $5(8.8)$ & $1(10.0)$ \\
\hline Only if it hurts & $32(56.1)$ & $4(40.0)$ \\
\hline \multicolumn{3}{|l|}{12} \\
\hline Closed & $88(58.7)$ & $16(59.3)$ \\
\hline Flat shoes & 20 (13.3) & $2(7.4)$ \\
\hline Boots & $34(22.7)$ & $5(18.5)$ \\
\hline Sandals & 29 (19.3) & $3(11.1)$ \\
\hline Sneakers & $53(35.3)$ & $12(44.4)$ \\
\hline Other & $9(6.0)$ & 0 \\
\hline 12-rec & $121(80.7)$ & $24(88.9)$ \\
\hline Closed & $29(19.3)$ & $3(11.1)$ \\
\hline Open & & $14(58.3)$ \\
\hline \multicolumn{3}{|l|}{13} \\
\hline Shoelaces & $81(58.7)$ & \\
\hline Buckle & $7(5.1)$ & $1(4.2)$ \\
\hline Velcro & $29(21.0)$ & $4(16.7)$ \\
\hline Zipper & $22(15.9)$ & $2(8.3)$ \\
\hline None & $37(26.8)$ & $4(16.7)$ \\
\hline \multicolumn{3}{|l|}{ 14-rec } \\
\hline Plain-low & 96 (73.3) & $19(95.0)$ \\
\hline Medium-high & $35(26.7)$ & $1(5.0)$ \\
\hline
\end{tabular}

Podiatric habits by pathology (ONC and ONM) are shown in table 2 . Of the people with ONC, $71.3 \%$ trim their nails themselves, with $37.7 \%$ using a nail clipper. In case of suffering from callosities of patches of hard skin, the most widely used method is pumice stone (29.5\%). Most usual footwear is closed $(58.7 \%)$, and the most common fastening system is with shoelaces $(58.7 \%)$, with flat heel being most widely used (44.7\%). As for those with ONM, $80.8 \%$ trim their nails themselves, and the most widely used method is nail clipper (34.6\%). In they suffer from callosities or patches of hard skin, $35 \%$ does nothing to solve it. Most widely used footwear is closed $(59.3 \%)$, with fastening by means of shoelaces $(58.3 \%)$ and with plain heel $(47.6 \%)$.

When a bivariate analysis was performed to determine if age, localization and number of localizations predict the occurrence of ONM, we found out that none of these variables has an independent effect to predict this pathology (Table 3).

To describe which variables can be predictors of ONM, a bivariate analysis was performed according to the studied subjects' habits (Table 4). In addition, a logistic regression bivariate analysis was carried out in order to establish if podiatric habits 1 ("Is this your
Table 3. Bivariate analysis to predict onychomycosis according to the age and localizations number variables

\begin{tabular}{|c|c|c|c|}
\hline & \multicolumn{2}{|c|}{ Onychomycosis } & \multirow[t]{3}{*}{$p$} \\
\hline & $\begin{array}{c}\text { Yes } \\
(n=140)\end{array}$ & $\begin{array}{c}\text { No } \\
(n=30)\end{array}$ & \\
\hline & Mean \pm SD & Mean \pm SD & \\
\hline Age & & & 0.180 \\
\hline \multirow[t]{2}{*}{$\begin{array}{l}\text { Number of onychocryptosis } \\
\text { localizations }\end{array}$} & $1,57 \pm 0,68$ & $1,57 \pm 0,61$ & 0.841 \\
\hline & n (\%) & n (\%) & \\
\hline $\begin{array}{l}\text { Localizations } \\
\text { Right foot }\end{array}$ & & & \\
\hline $\begin{array}{l}\text { First toe } \\
\text { Other toes } \\
\text { Left foot }\end{array}$ & 22 (73.3) & $8(26.7)$ & 0.849 \\
\hline $\begin{array}{l}\text { First toe } \\
\text { Other toes } \\
\text { Both feet first toe }\end{array}$ & $22(73.3)$ & $8(26.7$ & 0.833 \\
\hline
\end{tabular}

Means were compared using Student's t-test for the age and Mann-Whitney U-test for the number of onychocryptosis and onychomycosis localizations. The association between categorical variables is established by means of the chi-square test. SD: standard deviation.

Table 4. Bivariate analysis to predict onychomycosis according to patient habits

\begin{tabular}{|c|c|c|c|}
\hline \multirow[t]{3}{*}{ Habits } & \multicolumn{2}{|c|}{ Onychomycosis } & \multirow[t]{3}{*}{$p$} \\
\hline & $\begin{array}{c}\text { Yes } \\
(n=30)\end{array}$ & $\begin{array}{c}\text { No } \\
(n=140)\end{array}$ & \\
\hline & $\mathrm{n}(\%)$ & n (\%) & \\
\hline$\overline{1}$ & & & 0.765 \\
\hline No & $12(50.0)$ & $56(46.7)$ & \\
\hline Yes & $12(50.0)$ & $64(53.3)$ & \\
\hline 2 & & & 0.644 \\
\hline Less than half hour & $11(40.0)$ & $40(34.5)$ & \\
\hline 1 hour & $8(32.0)$ & $50(43.1)$ & \\
\hline Between 2 and 3 hours & $3(12.0)$ & $17(14.7)$ & \\
\hline 4 hours or longer & $3(12.0)$ & $9(7.8)$ & \\
\hline 3 & & & 0.917 \\
\hline Sitting & $8(32.0)$ & $35(29.4)$ & \\
\hline Standing up/short walks & $15(60.0)$ & $61(51.3)$ & \\
\hline Standing up/long walks & $2(8.0)$ & $25(21.0)$ & \\
\hline 4 & & & 0.028 \\
\hline No & $5(35.7)$ & $29(37.2)$ & \\
\hline Yes & $9(64.3)$ & $49(62.8)$ & \\
\hline 5-rec & & & 0.028 \\
\hline Yourself or a professional & 25 (96.2) & $96(77.4)$ & \\
\hline Non-professional & $1(3.8)$ & $28(22.6)$ & \\
\hline 6 & & & \\
\hline Nail scissors & $6(23.1)$ & $37(29.6)$ & \\
\hline Nail clip & $9(34.6)$ & $48(38.4)$ & \\
\hline Nail pliers & $8(30.8)$ & $30(24.0)$ & \\
\hline File & $2(7.7)$ & $19(15.2)$ & \\
\hline 7 & & & \\
\hline No & $17(70.8)$ & $65(52.8)$ & \\
\hline Yes & $7(29.2)$ & $58(47.2)$ & 0.105 \\
\hline
\end{tabular}


Table 4. Bivariate analysis to predict onychomycosis according to patient habits (Continued)

\begin{tabular}{|c|c|c|c|}
\hline \multirow[t]{3}{*}{ Habits } & \multicolumn{2}{|c|}{ Onychomycosis } & \multirow[t]{3}{*}{$p$} \\
\hline & $\begin{array}{c}\text { Yes } \\
(n=30)\end{array}$ & $\begin{array}{c}\text { No } \\
(n=140)\end{array}$ & \\
\hline & n (\%) & n (\%) & \\
\hline \multicolumn{4}{|c|}{8} \\
\hline Everyday & 3 (42.9) & 19 (30.2) & \\
\hline Every 2-3 days & $1(14.3)$ & $24(38.1)$ & \\
\hline Once a week & $1(14.3)$ & $16(24.5)$ & \\
\hline Once a month & $2(28.6)$ & $4(6.3)$ & \\
\hline \multicolumn{4}{|l|}{9} \\
\hline More than once daily & $2(8.7)$ & $27(22.5)$ & \\
\hline Once daily & $15(65.2)$ & $63(52.5)$ & \\
\hline 2-3 times a week & $4(17.4)$ & 27 (22.5) & \\
\hline Once a week & $2(8.7)$ & $2(1.7)$ & \\
\hline $\begin{array}{l}\text { Less than once a week } \\
\text { Other }\end{array}$ & $0(0.0)$ & $1(0.8)$ & \\
\hline \multicolumn{4}{|l|}{10} \\
\hline Callous treatments & $0(0.0)$ & $11(10.8)$ & \\
\hline Blades & $1(5.0)$ & 17 (16.7) & \\
\hline Pumice stone & $5(25.0)$ & $31(30.4)$ & \\
\hline Visit the podiatrist & $5(25.0)$ & $26(25.5)$ & \\
\hline Nothing & $7(35.0)$ & $23(22.5)$ & \\
\hline \multicolumn{4}{|l|}{11} \\
\hline Once a month & $1(10.0)$ & $10(21.3)$ & \\
\hline More than twice a year & $4(40.0)$ & $5(10.6)$ & \\
\hline Once a year & $1(10.0)$ & $4(8.5)$ & \\
\hline Only if it hurts & $4(40.0)$ & $28(59.6)$ & \\
\hline 12 & & & 0.017 \\
\hline Closed & $24(88.9)$ & $97(78.9)$ & \\
\hline Open & $3(11.1)$ & $26(21.1)$ & \\
\hline \multicolumn{4}{|l|}{13} \\
\hline Shoelaces & 14 (58.3) & $67(58.8)$ & \\
\hline Buckle & $1(4.2)$ & $6(5.3)$ & \\
\hline Velcro & $4(16.7)$ & $25(21.9)$ & \\
\hline Zipper & $2(8.3)$ & $20(17.5)$ & \\
\hline None & $4(16.7)$ & $33(28.9)$ & \\
\hline 14 & & & 0.017 \\
\hline Plain-low & $19(95.0)$ & $7(69.4)$ & \\
\hline Medium-low & $1(5.0)$ & 34 (30.6) & \\
\hline
\end{tabular}

* $86.4 \%$ of sneaker users claim to use plain-low heel, as well as $74.7 \%$ of closed footwear users.

first visit to the podiatrist?"), 2 ("During your daily activity, approximately how much do you walk?"), 4 (“Do you practice any kind of sport or hobby that involves physical activity?"), 5 ("Who looks after your toenails care?"), 7 ("Do you apply moisturizing cream to your feet?"), 12 ("What are the characteristics of the footwear you use more often?") and 14 ("What type of heel has the footwear you use more often?") predict the occurrence of ONM. Habits 5 and 14 were observed to have an independent effect to predict the occurrence of said pathology in the sense that attending a professional (podiatrist) is related to a higher
Table 5. Association of onychocryptosis localizations and footwear type (habit 12) and heel height (habit 14)

\begin{tabular}{|c|c|c|c|}
\hline \multirow[t]{2}{*}{ Habit 12} & \multicolumn{2}{|c|}{$\begin{array}{l}\text { Localization on left foot, } \\
\text { first toe }\end{array}$} & \multirow[t]{2}{*}{$p$} \\
\hline & Yes & No & \\
\hline \multirow{4}{*}{$\begin{array}{l}\text { Closed } \\
\text { Open }\end{array}$} & $85(77,3)$ & $36(90.0)$ & 0.081 \\
\hline & 25 (22.7) & $4(10.0)$ & \\
\hline & \multicolumn{2}{|c|}{$\begin{array}{l}\text { Localization on left foot, } \\
\text { other toes }\end{array}$} & \\
\hline & Yes & No & \\
\hline Closed & $7(77.8)$ & $114(80.9)$ & 0.821 \\
\hline \multirow[t]{3}{*}{ Open } & 2 (22.2) & 27 (19.1) & \\
\hline & \multicolumn{2}{|c|}{$\begin{array}{l}\text { Localization on right foot, } \\
\text { first toe }\end{array}$} & \\
\hline & Yes & No & \\
\hline Closed & $92(81.4)$ & $29(78.4)$ & 0.685 \\
\hline \multirow[t]{3}{*}{ Open } & $21(18.6)$ & $8(21.6)$ & \\
\hline & \multicolumn{2}{|c|}{$\begin{array}{l}\text { Localization on right foot, } \\
\text { other toes }\end{array}$} & \\
\hline & Yes & No & \\
\hline Closed & $3(37.5)$ & $118(83.1)$ & 0.001 \\
\hline \multirow[t]{3}{*}{ Open } & $5(62.5)$ & 24 (16.9) & \\
\hline & \multicolumn{2}{|c|}{$\begin{array}{c}\text { Bilateral localization, first } \\
\text { toe }\end{array}$} & \\
\hline & Yes & No & \\
\hline Closed & 58 (76.3) & $63(85.1)$ & 0.171 \\
\hline Open & $18(23.7)$ & $11(14.9)$ & \\
\hline
\end{tabular}

\begin{tabular}{|c|c|c|c|}
\hline \multirow[t]{2}{*}{ Habit 14} & \multicolumn{2}{|c|}{$\begin{array}{l}\text { Localization on left foot, } \\
\text { first toe }\end{array}$} & \\
\hline & Yes & No & \\
\hline \multirow{4}{*}{$\begin{array}{l}\text { Plain-low heel } \\
\text { Medium-high heel }\end{array}$} & $70(71.4)$ & $26(78.8)$ & 0.409 \\
\hline & $28(28.6)$ & $7(21.2)$ & \\
\hline & \multicolumn{2}{|c|}{$\begin{array}{l}\text { Localization on left foot, } \\
\text { other toes }\end{array}$} & \\
\hline & Yes & No & \\
\hline Plain-low heel & $5(62.5)$ & $91(74.0)$ & 0.477 \\
\hline Medium-high heel & $3(37.5)$ & $32(26.0)$ & \\
\hline
\end{tabular}

Localization on right foot

\begin{tabular}{llll} 
& \multicolumn{2}{c}{$\begin{array}{c}\text { Localization on right foot, } \\
\text { first toe }\end{array}$} & \\
\cline { 2 - 3 } & \multicolumn{2}{c}{ Yes } & No \\
\hline Plain-low heel & $72(74.2)$ & $24(70.6)$ & 0.680 \\
Medium-high heel & $25(25.8)$ & $10(29.4)$ & \\
\hline
\end{tabular}

Localization on right foot,

\begin{tabular}{lccc} 
& \multicolumn{2}{c}{$\begin{array}{c}\text { Localization on right foot, } \\
\text { other toes }\end{array}$} \\
\cline { 2 - 3 } & Yes & No & \\
\hline Plain-low heel & $6(85.7)$ & $90(72.6)$ & 0.445 \\
Medium-high heel & $1(14.3)$ & $34(27.4)$ & \\
\hline
\end{tabular}

(Continue) 
Table 5. Association of onychocryptosis localizations and footwear type (habit 12) and heel height (habit 14) (Continued)

\begin{tabular}{|c|c|c|c|}
\hline \multirow[t]{2}{*}{ Habit 12} & \multicolumn{2}{|c|}{$\begin{array}{l}\text { Localization on left foot, } \\
\text { first toe }\end{array}$} & \multirow[t]{2}{*}{$p$} \\
\hline & Yes & No & \\
\hline & \multicolumn{2}{|c|}{$\begin{array}{l}\text { Bilateral localization, first } \\
\text { toe }\end{array}$} & \\
\hline & Yes & No & \\
\hline $\begin{array}{l}\text { Plain-low heel } \\
\text { Medium-high heel }\end{array}$ & $\begin{array}{l}46(69.7) \\
20(30.3)\end{array}$ & $\begin{array}{l}50(76.9) \\
15(23.1)\end{array}$ & 0.350 \\
\hline
\end{tabular}

probability of suffering from ONM. This relationship may be due to the fact that a complaint that drives seeking help from a podiatrist is having nail disorders, in the same way that people who do not seek a specialist's aid are less likely to be diagnosed with ONM. With regard to habit 14, people who use plain or low heels are less likely to suffer from ONM. This may be due to the fact that those who use plain or low heel are people who use closed shoes $(74.7 \%)$, and among them, $86.4 \%$ use sneakers. Habit 7 is in the limit of clinical significance, in the sense that adhering to this habit is associated with higher probability to suffer from ONM. The remaining habits studied in the bivariate analysis have no independent effect to predict the occurrence of ONM.

In the bivariate analysis looking to establish a possible association of ONC localization and footwear type, a borderline statistically significant association was found between the footwear type and left foot (first toe) ONC localization ( $p=0.081$ ) and, hence, using closed footwear is associated with the presence of ONC on this foot's first toe.

Finally, a bivariate analysis was also carried out in order to establish the association between ONM localization and footwear type and heel height (Table 6). The use of plain-low heels was found to be associated with the presence of ONM on left foot first toe, with no statistically significant association with the rest of ONM localizations being shown.

\section{Discussion}

Onychopathies account for approximately $10 \%$ of all skin conditions ${ }^{11,17}$ and, out of them, ONM represents about $50 \%{ }^{11}$. Different authors have tried to establish the prevalence of ONC. Lloyd and Brill ${ }^{18}$ claim that it affects $20 \%$ of people with any foot pathology, whereas Krausz ${ }^{19}$ points out that $61 \%$ of the population has some type of nail pathology, with ONC standing out above all of them. In the studied sample, an ONC
Table 6. Association of onychomycosis localizations and footwear type (habit 12) and heel height (habit 14)

\begin{tabular}{|c|c|c|c|}
\hline \multirow[t]{2}{*}{ Habit 12} & \multicolumn{2}{|c|}{ Localization on left foot, first toe } & \multirow[t]{2}{*}{ p } \\
\hline & Yes & No & \\
\hline Closed & 14 (93.3) & $107(79.3)$ & 0.190 \\
\hline \multirow[t]{3}{*}{ Open } & $1(6.7)$ & $28(20.7)$ & \\
\hline & \multicolumn{2}{|c|}{$\begin{array}{l}\text { Localization on left foot, other } \\
\text { toes }\end{array}$} & \\
\hline & Yes & No & \\
\hline Closed & $1(100)$ & $120(80.5)$ & 1.000 \\
\hline \multirow[t]{3}{*}{ Open } & 0 & $29(19.5)$ & \\
\hline & \multicolumn{2}{|c|}{$\begin{array}{l}\text { Localization on right foot, first } \\
\text { toe }\end{array}$} & \\
\hline & Yes & No & \\
\hline Closed & $11(78.6)$ & $110(80.9)$ & 0.835 \\
\hline Open & $3(21.4)$ & $26(19.1)$ & \\
\hline
\end{tabular}

\begin{tabular}{lccc}
\hline & \multicolumn{3}{c}{$\begin{array}{c}\text { Localization on right foot, other } \\
\text { toes }\end{array}$} \\
\cline { 2 - 3 } & Yes & No & \\
\hline Closed & $2(100)$ & $119(80.4)$ & 1.000 \\
Open & 0 & $29(19.6)$ & \\
\hline
\end{tabular}

\begin{tabular}{lccc}
\hline & \multicolumn{3}{c}{ Bilateral localization, first toe } \\
\cline { 2 - 3 } & Yes & No & \\
\hline Closed & $3(75.0)$ & $118(80.8)$ & 0.771 \\
Open & $1(25.0)$ & $28(19.2)$ & \\
\hline
\end{tabular}

\begin{tabular}{lccc}
\hline Habit 14 & \multicolumn{3}{c}{ Localization on left foot, first toe } \\
\cline { 2 - 3 } & Yes & No & \\
\hline Plain-low heel & $11(100)$ & $85(70.8)$ & 0.036 \\
Medium-high heel & 0 & $35(29.2)$ & \\
\hline
\end{tabular}

\begin{tabular}{lccc}
\hline & \multicolumn{3}{c}{$\begin{array}{c}\text { Localization on left foot, other } \\
\text { toes }\end{array}$} \\
\cline { 2 - 3 } & Yes & No & \\
\hline Plain-low heel & $1(100)$ & $95(73.1)$ & 1.000 \\
Medium-high heel & 0 & $35(26.9)$ & \\
\hline
\end{tabular}

\begin{tabular}{lccc}
\hline & \multicolumn{2}{c}{$\begin{array}{c}\text { Localization on right foot, first } \\
\text { toe }\end{array}$} & \\
\cline { 2 - 3 } & Yes & No & \\
\hline Plain-low heel & $9(90.0)$ & $87(71.9)$ & 0.214 \\
Medium-high heel & $1(10.0)$ & $34(28.1)$ & \\
\hline
\end{tabular}

\begin{tabular}{|c|c|c|c|}
\hline & \multicolumn{2}{|c|}{$\begin{array}{l}\text { Localization on right foot, other } \\
\text { toes }\end{array}$} & \\
\hline & Yes & No & \\
\hline \multirow{4}{*}{$\begin{array}{l}\text { Plain-low heel } \\
\text { Medium-high heel }\end{array}$} & $1(100)$ & $95(73.1)$ & 1.000 \\
\hline & 0 & $35(26.9)$ & \\
\hline & \multicolumn{2}{|c|}{ Bilateral localization, first toe } & \\
\hline & Yes & No & \\
\hline Plain-low heel & $2(100)$ & $94(72.9)$ & 1.000 \\
\hline Medium-high heel & 0 & $35(27.1)$ & \\
\hline
\end{tabular}


prevalence of $15.7 \%$ has been found, with the female gender and subjects younger than 65 years being more affected. This figure is very similar to that published for the Galician population older than 65 years $^{20}$. There is no unanimity on which gender is more affected by ONC. While García and Fernández ${ }^{4}$ demonstrate higher frequency in the female gender, other authors refer that it is more common in males ${ }^{8,21}$. In turn, ONM is regarded as the main cause of nail conditions in developed countries. In the past few years, it has experienced an increased prevalence, generating considerable expenses to the health system ${ }^{13}$. In our study, the prevalence of ONM in subjects with ONC was $17.6 \%$, which is consistent with previously published figures ${ }^{20}$, and it was most frequently localized on the first toe ${ }^{22}$. This result would support the thesis that ONM prevalence in subjects with ONC is not higher than in subjects without an ONC diagnosis, since the obtained figure is within the range described for the general population ${ }^{12}$.

It should be pointed out that we haven't found studies in the Spanish population that have determined the prevalence of ONM in people with ONC. The most similar data were found in a study where, by means of a literature review, the incidence of ONC was analyzed in patients treated with oral antifungals ${ }^{23}$. Other studies carried out in North America and Mexico have observed an ONC incidence ranging from 18 to $37 \%$ after antifungal treatment by oral route ${ }^{14,15}$.

With regard to podiatric habits, a study in subjects older than 65 years observed that $55 \%$ of them cleaned their feet daily, less than half used moisturizing cream, the majority looked after their nails by themselves and only $28.9 \%$ resorted to a podiatrist in the face of a problem of hyperkeratosis or helomas ${ }^{24}$. These figures are highly similar to those in the present study $(54.5 \%, 44.2 \%, 71.4 \%$ and $26.1 \%$, respectively), although in our case most subjects with ONC were younger than 65 years. In another study carried out among students of the Universidad Complutense de Madrid (Spain), the totality of participants stated having attended a podiatrist some time, and $72 \%$ of them moisturized their feet daily ${ }^{25}$.

Guidelines for correct hygiene and care of the nails and of feet in general have been published. In this sense, daily washing the feet with subsequent moisturizing cream application is advisable ${ }^{26,27}$. Sometimes, the use of a pumice stone for hard skin patches elimination, an emery board or metal nail file for correctly grinding down and shaping the nails, or regular visit to a podiatrist is suggested. In general terms, studies analyzing habits of foot hygiene and care are quite scarce; nevertheless, they are abundant in the diabetic population ${ }^{28}$.

As for footwear, we know that adequate footwear and in good state can help to maintain balance, avoid pain and contribute to general wellbeing and individual independence for daily life activities, and that, conversely, the use of inadequate or defective footwear that elicits repeated microtrauma or favors a humid environment, promotes the development of ONM and other lesions such as hyperkeratosis and helomas. In our work, the most widely used footwear was of the closed type, coinciding with other studies carried out in Spanish populations older than 60 years ${ }^{29}$. However, we don't know the footwear characteristics with regard to shape or material, which may have been narrow, insufficiently long or poorly-transpiring shoes, in which case they might be considered as of risk for developing the pathologies under study. Sneakers have been observed to be the most widely used footwear in college student populations, with percentages even higher than that obtained in our study population, probably owing to differences in the study subjects' mean ages in one case and the other ${ }^{30}$.

These results indicate a priori that the prevalence of ONM in people with ONC is not higher than that found in subjects not diagnosed with ONC. However, given the scarcity of studies relative to this condition, we believe it is necessary to keep investigating for a better understanding of such a common nail disorder.

\section{References}

1. Dunn JE, Link CL Felson DT. Prevalence of foot and ankle conditions in a multiethnic community sample of older adults. Am J Epidemiol. 2004;159:491-8.

2. Munro BJ, Steele JR. Foot-care awareness: a survey of persons aged 65 years and older. J Am Podiatr Med Assoc. 1998;88:242-8.

3. Pita Fernández S, González Martín C, Seoane Pillado T, et al. Podiatric medical abnormalities in a random population sample 40 years or older in Spain. J Am Podiatr Med Assoc. 2014;104:574-82.

4. García Carmona FJ, Fernández Morato D. Tratamiento quirúrgico de la onicocriptosis. Madrid: Aula Médica; 2003.

5. Novel Martí V. Cirugía ungueal. En: Izquierdo Cases JO, editor. Podología quirúrgica. Madrid: Elsevier; 2006. p. 41-53.

6. García Carmona FJ, Padín Galea JM, Fernández Morato D. Onicocriptosis crónica. Piel. 2005;20:53-5.

7. Martínez Nova A. Podología: atlas de cirugía ungueal. Madrid: Panamericana; 2006.

8. Zavala Aguilar KZ, Gutiérrez Pineda F, Bozalongo de Aragón E. Manejo de la onicocriptosis en atención primaria. Caso clínico. Semergen. 2013;39:38-40.

9. Moreno Arroyo V, Granados Roldán MO, Tejera Vaquerizo AM. Onicocriptosis: un nuevo método terapéutico. Enferm Dermatol. 2009;3:36-7.

10. Fernández Arista G, Enríquez Merino J. Onicocriptosis: estudio comparativo del periodo posoperatorio de una matricectomía parcial lateral con el de una matricectomía parcial lateral con fenolización. Dermatol Rev Mex. 2006:50:87-93.

11. Domingo Herranz H, Ribera Pibernat M. Onicopatías más frecuentes en atención primaria. Jano. 2005;69:49-59. 
12. Larruskain Garmendia J, Idígoras Viedma P, Mendiola Arza J. Onicomicosis: diagnóstico y tratamiento. Información Terapéutica del Sistema Nacional de Salud. 2008;32:83-92.

13. Ballesté R, Mousqués N, Gezuele E. Onicomicosis: revisión del tema. Rev Med Urug. 2003;19:93-106.

14. Connelley Jr LK, Dinehart SM, McDonald R. Onychocryptosis associated with the treatment of onychomycosis. J Am Podiatr Med Assoc. 1999:89:424-6

15. Bonifaz A, Paredes V, Fierro L. Onychocryptosis as consequence of effective treatment of dermatophytic onychomycosis. J Eur Acad Dermatol. 2007;21:699-700.

16. Weaver TD, Jespersen DL. Multiple onychocryptosis following treatment of onychomycosis with oral terbinafine. Cutis. 2000;66:211-2.

17. Mosquera Fernández A, Bouza Vivero MC, Lluch Fruns J, et al. Abordaje conservador de la onicocriptosis leve. Enferm Dermatol. 2010:11:41-4.

18. Lloyd Davies RW, Brill GC. The aetiology and out-patient management of ingrowing toe-nails. Br J Surg. 1963;50:592-7.

19. Krausz CE. Nail survey (1942-1970). Br J Chirop. 1970;35:117.

20. Romero Soto M, Santalla Borreiros F, Gil Manso P, et al. Estudio de prevalencia de patologías podológicas y grado de dependencia en personas mayores de 65 años. Peu. 2010;30:104-11.
21. Murray WR, Bedi BS. The surgical management of ingrowing toenail. $\mathrm{Br}$ J Surg. 1975;62:409-12.

22. Nazar JR, Gerosa PE, Díaz OA. Onicomicosis: epidemiología, agentes causales y evaluación de los métodos diagnósticos de laboratorio. Rev Argent Microbiol. 2012;44:21-5.

23. Corral Lizana N, Álvarez Arroyo J. Relación entre el uso de antimicóticos y el aumento en la incidencia de onicocriptosis. Reduca. 2012;4:11.

24. Santalla Borreiros F, Romero Soto M, Gil Manso P, et al. Hábitos de cuidados podológicos en personas mayores de 65 años. Peu. 2010;30:36-41.

25. Cañas López MV, Rodríguez Olvera D. ¿Qué llevas en los pies? Reduca. $2014 ; 6: 34-8$

26. Gómez Ayala AE. Cuidados podológicos básicos. Farmacia Profesional. 2006;20:43-7.

27. Azcona Barbed L. Cuidados del pie. La salud en la base. Farmacia Profesional. 2004;18:58-62.

28. Fustero I. Cuidado de los pies. Offarm. 2007;26:66-72

29. Álvarez-Calderón Iglesias O, Alonso Tajes F, López López D, et al. Análisis del calzado en una población mayor de 60 años. Rev Int Cienc Podol. 2008:2:19-26.

30. Carlier Espeleta B, Santafé Martín C, Solano Alarcón R. Hábitos podológicos en una población universitaria. Reduca. 2011;3:5-6. 